\title{
On Wavelet Transform: An extension of Fractional Fourier Transform and its applications in optical signal processing
}

\author{
$\underline{\text { Vidya Sharma }}^{a}$ and Bharat Bhosale \\ ${ }^{a}$ Head, Dept. Of Mathematics, Arts, Commerce and Science College, Amravati, (MS), India. \\ ${ }^{b}$ Principal, Prof. Sambhajirao Kadam Mahavidyalaya, Deur, Tq. Koregaon, Dist. Satara (MS), India.
}

\begin{abstract}
Wavelet theory is associated with building a model for a signal, system or processes with a set of special signals and is emerged as a powerful tool of signal de-noising. Earlier, the much celebrated fractional Fourier transform (FrFT) has been used to decompose the contaminated signals and obtain the desired signals on removing the noise. In optical data transmission, continuous signals are represented as functions of space or spatial frequency. Fractional Fourier transforms are closely related to chirp transforms, which in turn characterize and formulate optical transmission as the propagation in free space whereas fractional domains are the generalizations of conventional space and frequency domain. This leads to an interpretation of fractional Fourier or chirp transforms as wavelet transforms. The Wavelet Transform of one dimension has two parameters viz. scaling and shifting parameters. This makes possible to establish the correspondence between fractional Fourier transform and wavelet transform by choosing chirp function as the wavelet transform kernel.

In this paper, a strong relationship between wavelet transform with fractional Fourier transform has been exploited to develop a full-fledged analytical framework in tempered distributional settings, which can be viewed as the extension of the FrFT. This interpretation of wavelet transform in terms of fractional Fourier transform is then used in filtering and separation of undesired noise and distortion from optical signals.

The proposed model has far reaching applications especially in the field of signal processing and in particular, in the field of long range optical fiber transmission; which has been an active area of research ever since the introduction of multiresolution techniques in the fractal representation of modulated signals.
\end{abstract}

Experimental results have shown that the wavelet based models have better performance over the other transform techniques ever applied for signal processing.

Keywords: Fractional Fourier transform, wavelet transform, signal processing, multiresolution 
Sharma and Bhosale, On Wavelet Transform: An extension of Fractional Fourier Transform and its applications in optical signal processing

\section{INTRODUCTION}

The concept of "Wavelets" started to appear in the literature only in the early 1980's. Morlet (1982), a French geophysical engineering, first introduced the idea of Wavelet Transform (WT) as a new mathematical tool for seismic signal analysis. The Wavelet transform decomposes a signal into a representation that shows signal details and trends as a function of time. This representation can be used to characterize transient events, reduce noise, compress data and perform many other operations. Wavelet transform have become a powerful tool of non stationary signal of various kinds such as sound, radar, sonar, seismic biomedical signals and for image processing, image compression and pattern recognition by Lokenath (1998).

Before the advent of wavelet transform, Kumar et. al. (2015) shown that the Fractional Fourier transform (FrFT) was most commonly used in areas of signal processing such as repeated filtering, fractional convolution and correlation, beam forming, optimal filter, convolution, filtering and wavelet transform, time frequency representation. A comprehensive mathematical frame work on properties and applications of FrFT can be found in Ozaktas et. al. (2000). The kernel of the FrFT is seen closely related to a particular class of wavelet family. This leads to the extension to wavelet transform.

\section{THE FRACTIONAL FOURIER TRANSFORM (FrFT)}

We introduced notion of FrFT which may be considered as fractional power of classical Fourier transform. The one dimensional fractional FT with parameter $\alpha$ of $f(x)$ denoted by $R^{\alpha} f(x)$ Young (1995), performs a linear operation, given by the integral transform

$\left[R^{\alpha} f(x)(\xi)=F_{\alpha}(\xi)=\int_{-\infty}^{\infty} K_{\alpha}(x, \xi) f(x) d x\right.$,

where the kernel $K_{\alpha}(x, \xi)=\frac{e^{i \alpha / 2}}{\sqrt{2 \pi i \sin \alpha}} \exp \left[\frac{i}{2 \sin \alpha}\left(\left(x^{2}+\xi^{2}\right) \cos \alpha-2 x \xi\right)\right]$

$$
=C_{1 \alpha} \exp \left(C_{2 \alpha}\left(\left(x^{2}+\xi^{2}\right) \cos \alpha-2 x \xi\right),\right.
$$

where $C_{1 \alpha}=(2 \pi i \sin \alpha)^{-1 / 2} \exp (i \alpha / 2)$ and $C_{2 \alpha}=\frac{1}{2} \sin \alpha$

The kernel, $K_{\alpha}(x, \xi)$ is the propagator of the non-stationary Schrodinger equation for a harmonic oscillator specified by Alieva (1994), which is well known in quantum mechanics (where $\alpha=\omega t$ relates to time $\mathrm{t}$ and classical frequency $\omega$, and $\xi$ is a position at the moment $t$ ).

\section{THE WAVELET TRANSFORM}

The classical wavelet transform of a function ' $\mathrm{f}$ ' with respect to a given admissible mother wavelet ' $\mathrm{g}$ ' is defined on wavelet domain coefficients at scale a and translation $b$ :

$$
\begin{aligned}
\left(W_{g} f\right)(a, b) & =|a|^{-1 / 2} \int f(x) g^{*}((x-b) / a) d x \\
& =f,|a|^{-1 / 2} g^{*}\left(\frac{x-b}{a}\right)=\left(f, g_{a, b}\right)=<f, U(a, b)>,
\end{aligned}
$$

* is a complex conjugate. Note here that integral function is on independent variable $\mathrm{x}$ and is mapped to 2dimensional function across scale and translator $\mathrm{a}$ and $\mathrm{b}$ respectively.

In n-dimensional set up, the continuous WT of $\varnothing$ with respect to the wavelet $\Psi$ is defined by

$(W \emptyset)(a, b)=\emptyset^{\wedge}(a, b)=\int_{R^{n}} \emptyset(x) \Psi *((x-b) / a) d x / a^{n}$

Provided that the integral exists, where $b \in R^{n}$ and $a>0$.

A reconstruction formula for (4) is given by

$$
\begin{array}{r}
\emptyset(x)=W^{-1}[\varnothing(a, b)](x)=\left(C_{\Psi}\right)^{-1} \int_{R_{+}} \int_{R^{n}} \emptyset(a, b) \Psi *((x-b) / a) d a d b / a^{n+1} \\
\text { where } C_{\Psi}=\int_{R^{n}}\left|\Psi^{*}(w)\right|^{2}|(\omega)|^{-n} d \omega>0
\end{array}
$$


Sharma and Bhosale, On Wavelet Transform: An extension of Fractional Fourier Transform and its applications in optical signal processing

It has been proved by Perrier and Bassdevant (1996) that for $\psi$ satisfying the admissibility condition (6) with $n=1$, the WT is a linear bounded operator

$W: L^{p}(R) \rightarrow L^{p}\left(R, L^{2}\left(R_{+}, \frac{d a}{a}\right)\right)=W^{p}, 1<p<\infty$,

Note that for the special case when the scale-translation surface is the wavelet transform of $\mathrm{f}$ with respect to mother wavelet $g$, then; $W^{-1}: W_{g}(a, b) \rightarrow f(x)$

The WT is a one-to-one operator only wavelet transform (wave domain distribution) exists for a given signal. However, it should be noted that the wavelet domain representation of a signal is not unique with respect to the same mother wavelet by Young (1995).

The signal $f \in L^{2}(R)$ can be uniquely represented in wavelet expansion, $f=\sum_{j, k} c_{j, k}(f) \psi_{j, k}$,

where $c_{j, k}(f)=\left\langle f, \psi_{j, k}\right\rangle$ are the wavelet coefficients and $\psi_{j, k}$ is a family of dilated and translated functions $\left\{\psi_{j, k}=2^{j / 2} \psi\left(2^{j},-k\right): j, k \in Z\right\}$, generated by the mother wavelet $\psi \in L^{2}(R)$.

\section{WAVELET TRANSFORM AS THE EXTENSION OF FrFT}

The kernel of the FrFT is closely related to Wavelet transform. Using straightforward algebraic manipulations and making the change of variable $b=\xi \sec \alpha$, we can write the FrFT of $f(x), f(x) \in$ $S^{*}\left(R^{n}\right)$

$$
\begin{aligned}
& {\left[R^{\alpha} \phi\right]\left(\frac{b}{\sec \alpha}\right)=A \frac{1}{|\alpha|^{n}} \int_{R^{n}} \phi(x) \exp \left[i \pi\left(\frac{x-b}{a}\right)^{2}\right] d x} \\
& g(b)=\left[R^{\alpha} f\right]\left(\frac{b}{\sec \alpha}\right)=A \frac{1}{|a|^{n}} \int_{R^{n}} f(x) \exp \left[i \pi\left(\frac{x-b}{\tan ^{\frac{1}{2}} \alpha}\right)^{2}\right] d x,
\end{aligned}
$$

$$
\text { where } A=\frac{C(\alpha)|a|^{n}}{\exp \left(\frac{i}{2}\right) b^{2} \sin ^{2} \alpha}, C(\alpha)=\frac{\exp \left(\frac{\alpha i}{2}\right)}{(2 \pi i \sin \alpha)^{\frac{1}{2}}}
$$

Taking $a=\tan ^{\frac{1}{2}} \alpha$, as scaling parameter and $b=\xi \sec \alpha$, as shifting parameter, the convolution represented by the above integral is a wavelet transform in which the wavelet family is obtained from the quadratic phase function $\psi(x)=\exp \left(i \pi x^{2}\right)$ by scaling the coordinate and the amplitude by $\tan ^{\frac{1}{2}}(\propto)$ and $C(\propto)$ respectively. $g(b)=\left[R^{\alpha} f\right]\left(\frac{b}{\sec \alpha}\right)=A .(W f)(a, b), f(x) \in S^{*}\left(R^{n}\right)$

Thus, the FrFT kernels corresponding to different values of $\alpha$ are closely related to a wavelet family.

Alternatively, we can write wavelet transform in terms of fractional Fourier transform as an obvious extension as,

$$
(W f)(a, b)=\frac{1}{A}\left[R^{\alpha} f\right]\left(\frac{b}{\sec \alpha}\right), f(x) \in S^{*}\left(R^{n}\right)
$$

The Generalized Wavelet transform as the extension of fractional Fourier transform of $f(x) \in S^{*}\left(R^{n}\right)$ is written as

$$
\begin{aligned}
& (W f)(a, b)=B\left[R^{\alpha} f\right]\left(\frac{b}{\sec \alpha}\right)=\left\langle f(x), K_{\alpha}(x, \xi)\right\rangle, \\
& \text { where } K_{\alpha}(x, \xi)=B C_{1 \alpha} e^{i C_{2 \alpha}\left[\left(x^{2}+\xi^{2}\right) \cos \alpha-2 x \xi\right]} \\
& \text { and } B=\frac{\exp \left(\frac{i}{2}\right) b^{2} \sin ^{2} \alpha}{C(\alpha)\left|\alpha^{n}\right|}, C(\alpha)=\frac{\exp \left(\frac{\alpha i}{2}\right)}{(2 \pi i \sin \alpha)^{\frac{1}{2}}}, \xi=\frac{b}{\sec \alpha} \\
& \qquad C_{1 \alpha}=(2 \pi i \sin \alpha)^{-1 / 2} \exp (i \alpha / 2), C_{2 \alpha}=\frac{1}{2} \sin \alpha
\end{aligned}
$$

The right hand side of equation (8) is indeed meaningful since for each $\xi \in R^{n}$ and $0<\alpha \leq \frac{\pi}{2}$, the function $K_{\alpha}(x, \xi)$ belongs to $S\left(R^{n}\right)$ as a function of $x$, 
Sharma and Bhosale, On Wavelet Transform: An extension of Fractional Fourier Transform and its applications in optical signal processing

$$
\begin{aligned}
\gamma_{v, \beta}\left(K_{\alpha}(x, a, b)\right) & =\sup _{x \in R^{n}}\left|(1+|x|)^{v} D_{x}^{\beta}(x) K_{\alpha}(x, a, b)\right| \\
& =\sup _{x \in R^{n}}\left|(1+|x|) D_{x}^{\beta}(x) B C_{1 \alpha} e^{i C_{2 \alpha}\left[\left(x^{2}+\xi^{2}\right) \cos \alpha-2 x \xi\right]}\right| \\
& =\sup _{x \in R^{n}}\left|(1+|x|) B \sum_{h=0}^{m} C_{m} C_{\alpha}(x \cos \alpha-\xi)^{m-2 h} e^{(m-h) t}\right|, \\
\text { where } C_{m} & =\frac{m !}{(m-2 h) ! h !}(i)^{m-h}(2)^{m-2 h}, C_{\alpha}=\left(C_{2 \alpha}\right)^{m-h} \cos ^{h} \alpha \\
t & =i c_{2 \alpha}\left[\left(x^{2}+\xi^{2}\right) \cos \alpha-2 x \xi\right. \\
& \quad \text { for any fixed } x \in R^{n} \text { and any fixed } m, 0<\alpha \leq \frac{\pi}{2} .
\end{aligned}
$$

\section{PHYSICAL INTERPRETATION OF WAVELET TRANSFORMS}

It has recently been shown that the formulation of optical diffraction can be viewed into a similar wavelet framework. In optical data transmission, continuous signals are represented as functions of space or spatial frequency. Also, we see that the fractional Fourier transforms are closely related to chirp transforms. Chirp transform in turn characterize and formulate optical transmission as the propagation in free space. This provides the basis of the concept of fractional domains, which are generalizations of the conventional space and frequency domains. This leads to an interpretation of fractional Fourier or chirp transforms as wavelet transforms.

Having interpreted the wavelet transform in terms of fractional Fourier transform, we now use this consideration in filtering and separation of undesired noise and distortion from optical signals.

Note that the wavelet transform of one dimension has two parameters viz. scaling and shifting parameters. It is possible to establish the correspondence between fractional Fourier transform say $f^{\alpha}(x)$, and wavelet transform by choosing chirp function as the wavelet transform kernel.

We illustrate the application of wavelet transform in de-noising corrupted signals.

\subsection{Wavelet De-noising}

In optical systems, transmitted signals are usually distributed partially, or sometimes almost completely by an additive noise from the transmitter, channel, and receiver. Noise arises due to imperfect instruments used in signal processing, problems with the data acquisition process, and interference which can degrade the data of interest. Also, noise can be introduced due to compression and transmission errors by Motwani et. al. (2004). De-noising or the noise reduction is an essentially required process to enhance the estimation process of signal/image reconstruction of the captured signal.

Wavelets localize features in given data to different scales, thus can preserve important signal or image features while removing noise by Bhosale (2012). The basic idea behind wavelet de-noising, or wavelet thresholding is that the wavelet transform concentrates signal and image features in a few large-magnitude wavelet coefficients. Wavelet coefficients which are small in value are typically noise and you can "shrink" those coefficients or remove them without affecting the signal or image quality. After thresholding the coefficients, the original signal can reconstructed applying inverse wavelet transform.

Wavelet de-noising is a simple operation, which aims at reducing noise in a noisy image. It is performed by selecting the wavelet coefficients below a certain threshold and setting them to zero as follows:

$$
\begin{aligned}
y_{\lambda} & =y_{\lambda}, y_{\lambda} \geq t_{\lambda} \\
& =0, y_{\lambda}<t_{\lambda}
\end{aligned}
$$

where $t_{\lambda}$ is the threshold and $\lambda$ is the index.

The threshold used is $t_{\lambda}=k \sigma_{\lambda} \sigma$, for some scale $k$, where $\sigma$ is an estimation of the standard deviation of the noise, $\sigma=\frac{\text { Median }}{0.6745}$, and $\sigma_{\lambda}$ is an approximation value for the standard deviation of each wavelet coefficient estimated by using the Monte-Carlo simulation. 
Sharma and Bhosale, On Wavelet Transform: An extension of Fractional Fourier Transform and its applications in optical signal processing

The following graphic shows the denoised signal through wavelet thresholding along with original signal

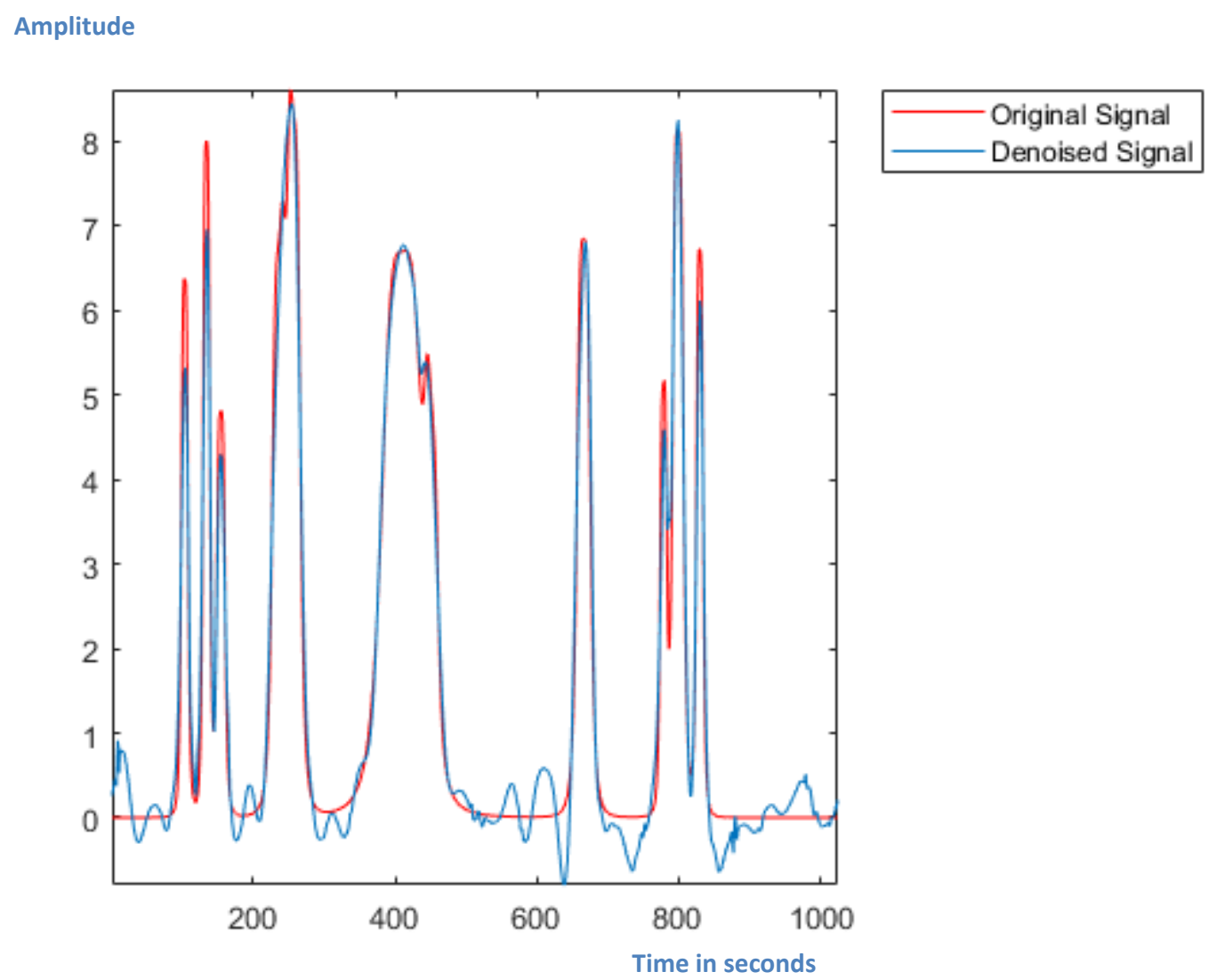

Figure 1. Original signal with denoised signal after applying wavelet thresholding

We can clearly see that wavelet denoising has removed a considerable amount of the noise while preserving the sharp features in the signal.

To conduct the performance factor analysis, the peak signal to noise ratio (PSNR) and root mean square error (RMSE) measures are used.

$$
P S N R=10 \log _{10}\left(\frac{\sum_{i=1}^{N} x^{2}(i)}{\sum_{i=1}^{N}[x(i)-\hat{x}(i)]^{2}}\right), R M S E=\frac{1}{N} \sqrt{\sum_{i=1}^{N}[x(i)-\hat{x}(i)]^{2}}
$$

where $x(i)$ is the original source signal, $\hat{x}(i)$ is the separated signal, $i$ is the sample index and $N$ is the number of samples of the signal.

The test is: Higher the value of PSNR with minimum value of RMSE, better the performance of the denoising model.

To illustrate the performance of the proposed wavelet based model, simulations are performed on noisy mixed optical signal data on Matlab ${ }^{\circledR}$ R 7.9 on a core i7 $2.2 \mathrm{GHz}$ PC using USFFT software package.

Algorithm: Apply the wavelet transform on sample signal

1. Parameters: Set $X$ and Bayesian classification

2. Initialization: $Y=\operatorname{SMOOTH}(X, S P A N)$ 
Sharma and Bhosale, On Wavelet Transform: An extension of Fractional Fourier Transform and its applications in optical signal processing

3. Decompose signal into sub bands $F_{j}$ with block size $S_{j}$

4. Apply the digital wavelet transform to each block to get wavelet coefficients at scale $j$

5. Apply threshold function choosing interval $\left[-t_{\lambda}, t_{\lambda}\right]$, the $f\left(c_{k}, t_{\lambda}\right) \rightarrow 0$ in $\left[-t_{\lambda}, t_{\lambda}\right]$, and $f\left(c_{k}, t_{\lambda}\right)=$ $c$, to demise the wavelet coefficients of input signal.

6. In the end, apply inverse wavelet transform to get the desired signal.

The results (PSNR in $\mathrm{dB}$ ) and corresponding RMSE for each sample data are presented below:

Table 1. PSNR and RMSE values for different sample data

\begin{tabular}{|l|l|l|l|l|}
\hline Images & Covariance & $\begin{array}{l}\text { Fractional } \\
\text { Fourier } \\
\text { transform }\end{array}$ & $\begin{array}{l}\text { Wavelet } \\
\text { thresholding }\end{array}$ & RMSE \\
\hline $\begin{array}{l}\text { Sample I } \\
\text { Random }\end{array}$ & $\mathrm{m}=0, \sigma^{2}=0.01$ & $\begin{array}{c}21.19(\mathrm{~m}=0, \\
\left.\sigma^{2}=0.01\right)\end{array}$ & 23.89 & 0.1433 \\
Noise) & $\mathrm{m}=0, \sigma^{2}=0.01$ & $\begin{array}{c}19.77(\mathrm{~m}=0, \\
\left.\sigma^{2}=0.01\right)\end{array}$ & 27.89 & 0.1234 \\
\hline $\begin{array}{l}\text { Sample II } \\
\text { (Gaussian } \\
\text { Noise) }\end{array}$ & & & \\
\hline
\end{tabular}

From the experimental results, it is seen that the wavelet based de-noising operation gives increased PNSR with minimum RMSE for sample image. Similar simulations can be performed for optical data, satellite images, seismic data.

\section{CONCLUSION}

In this work, the theory of fractional Fourier transform has been extended to wavelet transform by exploiting a strong relationship between Wavelet transform with fractional Fourier transform to develop a full-fledged analytical framework for wavelet transform in tempered distributional settings. The extended version of wavelet transform has far reaching applications especially in the field of signal processing and in particular, in the field of long range optical fiber transmission. Experimental results have shown that the wavelet based models have better performance over the other transform techniques ever applied for signal processing.

\section{REFERENCES}

Alieva, T. and Barbe A. (1997). Fractional Fourier analysis of objects with scaling symmetry in the book: Fractals in Engineering. ed. J. Lavy-Vehel, E. Lutton and C. Tricot, Springer Verlag. pp 252-265.

Alieva, T., Lopez V. and et. al (1994). J. Mod. Optics, 41 PP 1037-1044.

Bhosale B., Biswas A. (2012). Wavelet analysis of optical solitons and its energy aspects. J. Mathematics in Engineering, Science and Aerospace, 3, pp. 15-27.

Bhosale B., Biswas A. (2012). Wavelet analysis of soliton interaction and its relation to probability distributions. J. Nonlinear Studies, 19, pp. 563-572.

Kumar Rajneesh, Ahlawat Anil (June 2015). Removal of Image Blurriness using Fractional Fourier Transform. International Journal of Emerging Research in Management \&Technology, ISSN: 22789359, Volume-4, Issue-6.

Lokenath Debnath (Nov.1998). Wavelet transform and their applications. PINSA-A, 64,A, No.6, 685-713.

Morlet J, Arens G. et. al. (1982 a). J. Geophys 47, 203.

Miah K. H., Herrera R. van der Baan H., M., and Sacchi M. D. (2011). Application of Fractional Fourier Transform in Cepstrum Analysis. AAPG Search and Discovery Article \#90173 CSPG/CSEG/CWLS, GeoConvention 2011, Calgary, Alberta, Canada, May 9-11.

Motwani, M., Gadiya M., and Motwani R. (2004). Survey of image denoising techniques. Proceedings of global signal processing expo and conference, 27-30.

Ozaktas H.M., Zalevskys Z., Kutay M.A. (2000). The Fractional Fourier transform with applications in optics and signal and signal processing. Wiley, New York.

Perrier, V. and Basdevant C. (1996). Besov norms in terms of continous wavelet transform, Applications to structural functions. Math. Models Methods Appl. Sci., 6. 649-664. 
Sharma and Bhosale, On Wavelet Transform: An extension of Fractional Fourier Transform and its applications in optical signal processing

Sharma V. D., Deshmukh P. B. (June 2017). Poisson Summation Formula for Two Dimensional Fractional Fourier Transform. International Journal of Research in Engineering and Applied Sciences(IJREAS), Vol. 7, Issue 6, pp. 63 68.

Sharma V. D., Deshmukh P. B. (July 2017). Sampling Theorem Two Dimensional Fractional Fourier Transform. International Journal of Mathematical Archive, 8(7), pp 81-85.

Young, Randy K. (1995). Wavelet theory and its applications. Kluwer Academic Publishers. 